\title{
Utilização de modelos de regressão logística para a previsão de risco de liquidez em micro e pequenas empresas
}

Graduação em andamento em Administração pela Universidade Federal de Viçosa -

UFV

Av. PH Rolfs Campus Universitário. Viçosa/MG. CEP: 36570-000

E-mail: admlucasmaia@hotmail.com

Marco Aurélio Marques Ferreira Doutorado em Economia Aplicada pela Universidade Federal de Viçosa - UFV

Av. PH Rolfs Campus Universitário. Viçosa/MG. CEP: 36570-000

E-mail:marcoaurelio@ufv.br

Evandro Rodrigues de Faria Mestrado em Administração pela Universidade Federal de Viçosa - UFV

Av. PH Rolfs Campus Universitário. Viçosa/MG. CEP: 36570-000 E-mail: evandro_farias@yahoo.com.br

\section{RESUMO}

O presente estudo teve como objetivo investigar fatores limitantes da gestão de capital de giro em micro e pequenas empresas (MPEs) na cidade de Viçosa, MG. Para constatar a influência de variáveis na probabilidade de risco de liquidez, foi elaborado um modelo de regressão logística, em que a variável dependente foi construída pela identificação de problemas de capital de giro, a partir de variáveis condicionantes do risco de liquidez. Em seguida, aplicou-se o modelo logit que revelou um poder de previsão correta de $87,7 \%$, apresentando melhor desempenho para prever empresas classificadas sem risco de liquidez. A classificação mostrou-se eficiente para demonstrar a existência de variáveis condicionantes do risco de liquidez por meio dos testes de significância, possibilitando a elaboração de uma função de probabilidade.

Palavras-chave: Risco de liquidez. Regressão logística. Micro e pequenas empresas.

\section{An insight on the use of logistic model regression to predict the liquidity risk in} small companies

\section{ABSTRACT}

This paper aimed to investigate the restrictive factors of working capital management in micro and small business (MSBs) in the city of Viçosa, MG. To perceive the influence of the variable in the probability of liquidity risk, it was used a model of binary logistic 
Utilização de modelos de regressão logística para a previsão de risco de liquidez em micro e pequenas empresas

Lucas Maia dos Santos, Marco Aurélio Marques Ferreira, Evandro Rodrigues de Faria

regression analysis in which the subordinated variables was built through the identification of working capital problems. Then, the executed binary logistict regression model has obtained a correct prevision force in $87,7 \%$ of the cases, presenting a better development to forecasting businesses classified with no liquidity risk. The classification was efficient to show the existence of conditioning variables of liquidity risk by means of significance tests, allowing the preparation of a probability function.

Key Words: Liquidity risk. Binary logistic regression. Micro and small businesses.

\section{INTRODUÇÃO}

As micro e pequenas empresas (MPEs) compõem importante parcela da economia nacional e mundial. De acordo com o SEBRAE (2005), essas empresas, no Brasil, respondem por $99,2 \%$ do número total de empresas formais, por $57,2 \%$ do total de empregos e por $26 \%$ da massa salarial. Um dos papéis designados a elas é a oferta de produtos e serviços demandados pela sociedade, que só podem ser rentáveis se comercializados em pequena escala.

Apesar de serem um dos pilares da economia nacional, segundo o SEBRAE (2005), aproximadamente $60 \%$ das MPEs se extinguem até o quarto ano de existência. De acordo com recente estudo sobre fatores condicionantes e taxa de mortalidade das MPEs, realizado pelo SEBRAE (2007), os próprios empresários apontam problemas com carga tributária elevada como o principal fator para que a empresa encerrasse sua atividade, seguido por problemas na administração de capital de giro. A primeira razão apontada é externa à empresa, enquanto a outra é inerente à gestão financeira de curto prazo.

Em razão das suas peculiaridades, as MPEs enfrentam problemas bem específicos, em geral, diferentes ou em proporções distintas quando comparados àqueles vivenciados pelas grandes empresas. Às vezes as MPEs são atrativas do ponto de vista de rentabilidade, porém, devido às deficiências na gestão financeira de curto prazo, operam em alto risco de liquidez, o que as deixam suscetíveis a imprevistos comuns à natureza dos negócios. Isso instiga estudos voltados à análise da gestão de capital de curto prazo nessas empresas, como proposto neste estudo. 
Utilização de modelos de regressão logística para a previsão de risco de liquidez em micro e pequenas empresas

Lucas Maia dos Santos, Marco Aurélio Marques Ferreira, Evandro Rodrigues de Faria

Após investigar e questionar os proprietários de empresas extintas sobre os possíveis problemas que as levaram ao fracasso observou-se discrepância entre os motivos concretamente apontados por eles e os que consideravam, abstratamente, serem os principais problemas que afetaram suas empresas. Nessa situação, os empresários, em geral, são resistentes a enxergar distorções em sua forma de gestão. Em vez disso, culpam outras questões, como a inflação, a economia, a burocracia, as políticas públicas de crédito, a concorrência, entre outros, enquanto a autocrítica é relegada a último plano, o que remete à passividade por parte do empresário. Chér (1990) denomina esse problema de "efeito avestruz".

Por sua vez, problemas como esse impactam o desenvolvimento local e regional, em razão da grande empregabilidade desse setor, o que sugere ações de investigação das causas de limitação financeira, principalmente no que tange à gestão de capital de giro, apontada como grande fator de restrição entre essas organizações e que remete à forma de gestão do sócio-proprietário das MPEs. Resnik (1990) aponta como conseqüências das limitações financeiras: a perda da confiança e da moral do empresário; a elevação dos preços ou sua drástica redução em ato de desespero do empresário, colocando-os fora da realidade do mercado; a má remuneração de funcionários; o não pagamento de impostos; e a falta de fundos para sanar contingências.

Nesse contexto, o presente artigo teve como objetivo investigar os fatores limitantes da gestão de capital de giro, descrevendo os instrumentos, as ferramentas e as metodologias utilizadas nas micro e pequenas empresas do município de ViçosaMG.

\section{REFERENCIAL TEÓRICO}

\subsection{Fatores de limitação na gestão financeira de MPEs}

Segundo Braga et al. (2004), a falta de recursos financeiros é apontada como uma das maiores dificuldades com que convivem as MPEs. Este é um fator que limita os investimentos necessários para que essas empresas se desenvolvam ou, até mesmo, sobrevivam. 
Utilização de modelos de regressão logística para a previsão de risco de liquidez em micro e pequenas empresas

Lucas Maia dos Santos, Marco Aurélio Marques Ferreira, Evandro Rodrigues de Faria

De acordo com Monteiro (2003), as pequenas organizações possuem capacidade e recursos restritos, especialmente quanto ao capital (dificuldade para obtenção de empréstimos e fluxo de caixa reduzido) e à formação daqueles que atuam na administração e assessoria, cujas habilidades e informações necessárias ao planejamento estratégico adequado são, com freqüência, insuficientes. Assim, além do tamanho da empresa, também a atitude dos dirigentes configura-se como barreira ao processo de gestão financeira.

Matias e Lopes Júnior (2002) mostram que as dificuldades começam no dia-a-dia do proprietário, que, por falta de condições para contratação de pessoas eficientes e de confiança para a gestão do negócio, acaba acumulando funções, culminando no inadequado desempenho das atividades gerenciais. Cita ainda que a maior dificuldade das micro e pequenas empresas é o insuficiente preparo gerencial de proprietários ou dirigentes, destacando que as estratégias dessas empresas são estabelecidas confiando puramente em informações e experiências vivenciadas pelos seus administradores, confirmando um fator predominante que influencia diretamente as decisões financeiras de curto prazo.

Além dos problemas já citados, são listadas, a seguir, outras questões que dificultam a gestão financeira nas micro e pequenas empresas:

- Deficiência no planejamento prévio à abertura da empresa; má gestão; problemas particulares; problemas entre os sócios (Neves; Pessoa, 2006).

- A informalidade dificulta o mapeamento de indicadores de desempenho para a gestão financeira (Biléssimo, 2002).

- A contabilidade tradicional, muitas vezes única fonte de informação de micro e pequenas empresas, não fornece bases suficientes para a tomada de decisão (Gazzoni, 2003).

- A falta de recursos financeiros e dificuldades na obtenção de créditos, financiamentos $\mathrm{e}$ investimentos de terceiros, isto porque investidores raramente sentem-se atraídos por empresas de menor porte (Chér, 1990).

- A manutenção de maus pagadores, falta de disciplina, responsabilidade e organização (Neves; Pessoa, 2006). 
Utilização de modelos de regressão logística para a previsão de risco de liquidez em micro e pequenas empresas

Lucas Maia dos Santos, Marco Aurélio Marques Ferreira, Evandro Rodrigues de Faria

- A falta de controle, que ocasiona dificuldade para análise e avaliação de decisões tomadas, quando da exposição da empresa ao ambiente de negócios (GAZZONI, 2003).

O "efeito sanduíche", situação de perda do poder de barganha quando os preços de compra são impostos pelos fornecedores e os preços de venda pelos clientes, ocasionados pelo relacionamento freqüente com grandes fornecedores ou grandes clientes (Chér, 1990).

Acredita-se que esses fatores tendem a potencializar as limitações na gestão financeira destas empresas, com forte assimetria no que diz respeito às limitações de curto prazo, em que o reflexo mais observado tem sido o problema com capital de giro, ponto central deste estudo.

\subsection{A administração financeira de curto prazo nas MPEs}

É de conhecimento que nas micro e pequenas empresas não há clara diferenciação entre administração financeira e contabilidade. Além disso, a contabilidade não é encarada como instrumento da administração financeira, e sim como forma de atender às exigências legais e burocráticas.

Souza (2007) argumenta que não cabem ao empresário as funções de recolher tributos, de preencher guias para a Receita Federal, de recolher contribuições sociais, etc. Este trabalho deve ser delegado a empresas de assessoria contábil, de forma comum aos escritórios de contabilidade. Ao empresário cabe atuar como administrador financeiro, controlando estoques, contabilizando custos, elaborando planilhas para apurar seus resultados, decidindo sobre investimentos, financiamentos e planejando o fluxo de caixa. Quando necessário e possível, o empresário deverá buscar orientação profissional para a realização dessas tarefas.

Considerando que no ativo encontram-se as aplicações de curto prazo, Braga et al. (2004) entendem que o capital de giro se insere no contexto das decisões financeiras de curto prazo, envolvendo a administração de ativos e passivos circulantes.

Segundo Pereira Filho (1998) e Lemes Junior et al. (2005), o ativo circulante é composto por quatro componentes principais: caixa, títulos negociáveis, estoques e 
Utilização de modelos de regressão logística para a previsão de risco de liquidez em micro e pequenas empresas

Lucas Maia dos Santos, Marco Aurélio Marques Ferreira, Evandro Rodrigues de Faria

contas a receber. A gestão de contas a receber é parte de uma política de crédito e cobrança integrada com as metas de negócios da organização, envolvendo os termos de crédito e negociação de descontos a serem concedidos aos clientes. O volume de estoques deve ser tal que não se pode perder vendas por falta de estoque, porém, se for possível é preferível trabalhar sem estoque. Normalmente, contas a receber e estoques são os itens mais representativos do ativo circulante, de modo que outros valores a receber e despesas pagas antecipadamente tendem a não ser representativos em termos de valores. A manutenção de elevados ativos circulantes significa maior capacidade de pagamento da empresa, ou seja, maior liquidez. Por outro lado, os recursos aplicados no ativo circulante têm um custo de manutenção.

Braga et al. (2004) afirmam que o ativo circulante representa o valor dos recursos aplicados pela empresa para movimentar seu ciclo operacional, que compreende o período de tempo que vai desde a entrada da matéria-prima no estoque da empresa até a venda dos produtos elaborados e respectivo recebimento. Vários autores definem o ciclo operacional como o montante de tempo que vai do ponto em que a empresa coloca material e trabalho no processo de produção, até o momento em que o dinheiro da venda do produto acabado resultante é efetivamente recebido. $O$ ciclo operacional também pode ser deduzido pela soma do período médio de estocagem e recebimento dos clientes. Dentro do ciclo operacional encontra-se o ciclo de caixa, definido como montante de tempo em que o caixa da empresa está imobilizado entre o pagamento dos insumos de produção e a cobrança do pagamento da venda do produto acabado resultante (SOUZA, 1997, 2007; PEREIRA FILHO, 1998; LEMES JUNIOR et. al., 2005).

Pereira Filho (1998) afirma que paralelamente ao ciclo operacional ocorre o financiamento concedido pelos fornecedores, a partir do momento da compra. Até o momento do pagamento aos fornecedores a empresa não precisa se preocupar com o financiamento, o qual é automático. Se o prazo médio de pagamento de fornecedores (PMPF) for superior ao prazo médio de estoques (PME), então os fornecedores financiarão também uma parte das vendas da empresa. 
Utilização de modelos de regressão logística para a previsão de risco de liquidez em micro e pequenas empresas

Lucas Maia dos Santos, Marco Aurélio Marques Ferreira, Evandro Rodrigues de Faria

De acordo com Souza (2007), em geral as empresas seguem um ciclo no qual compram estoques, vendem mercadorias a crédito e depois cobram as contas a receber. A boa política de capital de giro é elaborada para minimizar o tempo entre desembolsos de caixa com materiais e recebimentos das vendas. Para esse autor, a disponibilidade de caixa deve existir pelas seguintes razões:

- Obter descontos dos fornecedores, tendo em vista que o custo de não utilizá-los é alto.

- Manter a classificação de crédito, por meio da liquidez corrente e sua liquidez seca alinhadas com as de outras empresas de seu setor.

- Aproveitar oportunidades de negócios favoráveis, como promoções especiais dos fornecedores ou a chance de adquirir outra empresa.

- Enfrentar emergências como greves, incêndios ou campanhas de marketing dos concorrentes e também para suportar quedas sazonais ou cíclicas no nível de atividade.

Toda empresa precisa buscar um nível satisfatório de capital de giro, de maneira a garantir a sustentação de sua atividade operacional. O equilíbrio financeiro é verificado quando suas obrigações financeiras se encontram lastreadas em ativos com prazos de conversão em caixa similares aos dos passivos; ele exige vinculação entre a liquidez dos ativos e os desembolsos demandados pelos passivos. Como afirmam Hopp e Leite (1989) e Pereira Filho (1998), para manter em equilíbrio financeiro uma opção da empresa é financiar suas necessidades variáveis com dívidas de curto prazo, utilizando os recursos de longo prazo para financiar todas as suas necessidades financeiras permanentes.

Muitos especialistas, incluindo Monteiro (2003), Gazzoni (2003) e Neves e Pessoa (2006), argumentam que as empresas "quebram" por terem problemas de liquidez, pois, se não houver dificuldade financeira, não há razão para quebrar. 0 principal objetivo da análise da liquidez de uma empresa reside em identificar sua capacidade de honrar seus compromissos de curto prazo. O relevante, entretanto, é identificar as causas que levam uma empresa a ter problemas de liquidez. O problema da dificuldade financeira decorre de ganhar pouco ou de gastar muito, ou ainda da 
Utilização de modelos de regressão logística para a previsão de risco de liquidez em micro e pequenas empresas

Lucas Maia dos Santos, Marco Aurélio Marques Ferreira, Evandro Rodrigues de Faria

combinação desses dois fatores. Entende-se, portanto, que a situação de liquidez de uma empresa é o efeito da combinação de diversos fatores de caráter operacional e de decisões estratégicas tomadas pela sua direção.

\section{METODOLOGIA}

\section{1 Área de estudo e amostra}

O presente estudo foi realizado no município de Viçosa - MG, localizado na Zona da Mata mineira, mais especificamente na microrregião da Mata de Viçosa. A escolha da região foi motivada pela alta concentração e representatividade das MPEs na economia local, principalmente aquelas ligadas ao comércio e à prestação de serviços. Para realização deste estudo foram entrevistados, pessoalmente, 172 proprietários de MPEs em funcionamento.

Para coleta de dados foram utilizadas as seguintes técnicas de pesquisa qualitativa em ciências sociais: pesquisa bibliográfica e aplicação de questionários. $\mathrm{Na}$ estratégia de coleta de dados utilizou-se amostra aleatória simples, estatisticamente significativa, com um erro máximo associado de aproximadamente $7,3 \%$. Na elaboração do questionário foi utilizada a técnica do balanço falado, citado por Kassai e Kassai (2001), que é um processo de planejamento empresarial para coleta de informações financeiras como uma alternativa mais próxima da realidade, em contrapartida aos relatórios financeiros elaborados por essas empresas.

Este estudo teve caráter exploratório e descritivo. Quanto à abordagem do problema e operacionalização das variáveis, a pesquisa foi quantitativa no levantamento e tabulação dos dados, e qualitativa na análise e comparação desses dados para identificar as especificidades do objeto de pesquisa, bem como os fatores limitantes da administração de capital de giro.

\subsection{Modelo de previsão de risco de liquidez}

Para perceber a influência de algumas variáveis na probabilidade de risco de liquidez, foi utilizado um modelo estatístico de probabilidade em que a variável 
Utilização de modelos de regressão logística para a previsão de risco de liquidez em micro e pequenas empresas

Lucas Maia dos Santos, Marco Aurélio Marques Ferreira, Evandro Rodrigues de Faria

dependente foi construída a partir da identificação de problemas de capital de giro, e estabelecida a partir de outras quatro variáveis. Para isso, a técnica do balanço falado abordada por Kassai e Kassai (2001) foi adaptada e aplicada em dois estágios. O primeiro foi constituído pelo questionamento direto ao empresário sobre a existência de problemas de capital de giro, e o segundo pela análise de questões recortadas em três dimensões: endividamento de curto prazo, ciclo de caixa e provisionamento de recursos de curto prazo.

A construção da variável problema de liquidez $Y$ surgiu a partir da análise da composição de outras quatro variáveis dicotômicas, denominadas $V_{i}(1 \leq i \leq 4)$, em que $V_{i}=0$ ou $V_{i}=1$. As variáveis $V_{i}$ assumiram os valores $V_{i}=1$ nas seguintes condições: empresários que citaram possuir falta de capital de giro $\left(V_{1}\right)$, endividamento de curto prazo superior a $90 \%\left(V_{2}\right)$, não-provisão para falta de caixa $\left(V_{3}\right)$ e ciclos de caixa superiores a 60 dias $\left(V_{4}\right)$. A existência de problemas de liquidez foi convertida para uma escala dicotômica, em que $Y=1$ representou a existência de problemas de liquidez, para somatório de $V_{i} \geq 2$, e $Y=0$ representou a inexistência de risco de liquidez, para somatório de $V_{i}<2$. Esse procedimento estabeleceu dois grupos de referência para análise neste estudo: empresas com risco de liquidez e sem risco de liquidez. Com esta classificação, aproximadamente $43 \%$ foram classificadas no grupo com risco de liquidez.

Em seguida, o modelo proposto foi construído com a utilização da técnica multivariada de regressão logística, conforme abordado em Pestana e Gageiro (2000), Scott (2002), Maroco (2003) e Harrel (2007). O objetivo da inclusão desse modelo como ferramenta empírica deste estudo foi determinar as variáveis que estão mais associadas à ocorrência de problemas de capital de giro, utilizado como proxy do risco de liquidez. Assim, não constituiu objetivo do estudo a identificação do efeito marginal daquelas sobre a variável dependente.

O Logit pertence à classe dos modelos estatísticos em que a variável explicada é, na verdade, uma variável qualitativa, ou, sendo quantitativa, dela apenas se pode observar manifestação de caráter qualitativo (OLIVEIRA, 2005). O objetivo desses modelos é refletir uma escolha entre duas alternativas, do tipo "sim ou não", "isto ou 
Utilização de modelos de regressão logística para a previsão de risco de liquidez em micro e pequenas empresas

Lucas Maia dos Santos, Marco Aurélio Marques Ferreira, Evandro Rodrigues de Faria

aquilo", "existe ou não existe" respostas estas marcadas pelo seu caráter de alta objetividade. "Os modelos mais simples dentro dessa classe são aqueles em que a escolha é uma ou outra de (apenas) duas alternativas disponíveis e mutuamente exclusivas" (OLIVEIRA, 2005: 326).

Como o problema remete à possível existência de variáveis categóricas, o teste de associação, bem como sua natureza, é realizado tomando como referência a distribuição de Qui-quadrado $\left(\chi^{2}\right)$, conforme procedimento destacado por Silver (2000). Nesse ponto, é oportuno destacar que, não havendo qualquer relação entre as variáveis independentes e a variável dependente, o valor de $\chi^{2}$ será nulo. Caso contrário precisa-se apenas perceber a diferença entre zero e o valor calculado, isto é, entre o valor observado e valor esperado, considerando se as variáveis independentes do estudo é algo mais que atribuíveis ao acaso ou erros de amostragem a dado nível de significância.

De acordo com Gujarati (2005), nesses modelos, a variável dicotômica é uma variável dummy, que assume os valores 0 ou 1, o que, na análise em questão, representa: 0 - caso a empresa não apresente problemas de capital de giro e 1 - caso a empresa apresente problemas de capital de giro.

No modelo Logit, define-se $P_{i}$ como a probabilidade de que a empresa apresente problemas de capital de giro. Essa probabilidade pode ser determinada por vários fatores (variáveis explicativas), representados por $X_{i}$, regredidos sobre a variável dependente $(\mathrm{Y})$. Dentre esses fatores, tem-se tempo de experiência do proprietário, faturamento bruto anual da empresa, número de empregados, entre outros.

Uma vez que a probabilidade é uma variável que se encontra no intervalo [0,1], a relação entre essa probabilidade e determinado atributo pode ser representada. No caso do modelo Logit, essa relação é representada pela função densidade de probabilidade acumulada logística. Tem-se, assim: $\boldsymbol{P}_{\boldsymbol{i}} \boldsymbol{f}\left(\boldsymbol{X}_{\boldsymbol{i}}\right)$, em que $f$ é uma função densidade probabilidade acumulada. 
Utilização de modelos de regressão logística para a previsão de risco de liquidez em micro e pequenas empresas

Lucas Maia dos Santos, Marco Aurélio Marques Ferreira, Evandro Rodrigues de Faria

Nesse modelo, a variável dependente da regressão é o logaritmo das chances de um evento específico, no caso o risco de liquidez, compreendido como o problema de capital de giro, conforme Equação 1:

$\mathrm{L}_{\mathbf{i}}=\ln \left(\frac{P_{i}}{1-P_{i}}\right)=X_{i} \beta$

Equação1

Assim, conforme descrito por Ferreira e Braga (2004), o lado esquerdo da equação 1 é denominado Logit, e é uma função linear dos parâmetros e das variáveis explicativas $\left(X_{i}\right)$. A probabilidade $P_{i}$ não é observável. Observa-se $\mathrm{y}=1$, se o evento ocorrer, e y $=0$, se o evento não ocorrer. Nesse sentido, a equação 1 deve ser ajustada pelo método de Máxima Verossimilhança e não por Mínimos Quadrados Ordinários (MQO), como maior parte das regressões lineares. Para maiores detalhes sobre 0 modelo recomenda-se Gujarati (2005).

Por meio da obtenção dos coeficientes das variáveis explicativas, pode-se perceber o impacto dessas na variação do logaritmo da probabilidade da variável dependente, aqui compreendida como risco de liquidez.

\subsubsection{Operacionalização das variáveis}

Esperava-se, a priori, que os fatores condicionantes relacionados à caracterização da estrutura, à administração, às características da gestão e do perfil gerencial das micro e pequenas empresas pudessem, em menor ou maior escala, refletir problemas na gestão do capital de giro.

A descrição da variável dependente, bem como as expectativas de relação das variáveis explicativas com o evento "ocorrência de problemas de capital de giro", são mais bem exploradas a partir da exposição a seguir.

Problemas de capital de giro $(\mathrm{Y})$ : Representa uma proxy de risco de liquidez, constituída por variáveis condicionantes do risco de liquidez. Assim, a empresa que declarou ter problemas de capital de giro tem como variável dependente $Y=1$, caso 
Utilização de modelos de regressão logística para a previsão de risco de liquidez em micro e pequenas empresas

Lucas Maia dos Santos, Marco Aurélio Marques Ferreira, Evandro Rodrigues de Faria

contrário, em que não foram declarados problemas de capital de giro foi atribuído $Y=0$ à variável dependente.

Número de empregados (EP): esta variável é um forte indicador de porte das organizações, por isso espera-se que números maiores de empregados estejam associados a menos problemas na gestão de capital de giro.

Número de sócios (SC): esta variável é adequada não só para quantificar os sócios da empresa, como também sua forma jurídica (empresário ou sociedade empresária limitada). Esperava-se que empresas com mais de um proprietário tivessem menos problemas com administração de capital, levando-se em conta aspectos como aumento do controle, ocasionado pela difusão do poder de decisão e diminuição do problema de agência, via maior transparência corporativa.

Setor (ST): esta variável tem aspecto qualitativo, sendo uma variável do tipo dummy; atribuiu-se 0 para indústria e 1 para comércio e serviços. Em geral, a atividade industrial requer maior complexidade, possuindo maiores barreiras de entrada. Isso requer das indústrias, estrutura e aporte superior de capital. Por isso, pressupunha-se que este setor estivesse ligado a menores probabilidades de problemas com capital de giro.

Percentual de capital próprio utilizado na abertura (CP): refere-se à fonte de financiamento inicial da empresa. Esperava-se que empresas com maior participação de capital próprio na abertura incorressem menos em problemas de capital de giro, porque o uso de fontes externas de financiamento tende a aumentar o risco da empresa, uma vez que encarece substancialmente o custo de capital.

Idade do sócio (IP): esperava-se que empresas cujos proprietários tivessem idade mais elevada enfrentariam menos problemas de gestão de capital de giro em razão da experiência acumulada ao longo dos anos.

Tempo de existência da empresa (TE): constitui o tempo total de atividade da organização, independente de registro jurídico. Era esperado que entre empresas com idade mais elevada os problemas de capital de giro fossem menos freqüentes por fatores como acesso facilitado a crédito, controles financeiros bem definidos, sólida participação de mercado, etc. 
Utilização de modelos de regressão logística para a previsão de risco de liquidez em micro e pequenas empresas

Lucas Maia dos Santos, Marco Aurélio Marques Ferreira, Evandro Rodrigues de Faria

Presença de investimentos (PI): variável que se refere à presença de investimentos no último ano. A presença de investimentos indica redução do caixa e provável aumento do risco de liquidez da empresa.

Faturamento Bruto Mensal (FB): é o principal indicador de porte para as organizações. Esperava-se neste trabalho que empresas com maior faturamento experimentassem menos problemas de gestão de capital de giro. Estas empresas são, em geral, mais sedimentadas no mercado. Além disso, possuem normalmente reservas financeiras e garantias reais, o que as tornam público de especial interesse para bancos.

Vendas a prazo (VP): o aumento do percentual de vendas a prazo aumenta 0 risco de inadimplência dos clientes e pode comprometer, no futuro, a capacidade de pagamento no curto prazo.

Prazo médio de recebimento (PMR): quanto maior o prazo médio de recebimento, maior o risco de liquidez no curto prazo.

Prazo médio pagamento (PMP): ao contrário do PMR, quanto menor o prazo de pagamento aos fornecedores, maior a probabilidade de risco de liquidez no curto prazo.

Percentual de caixa (PC): Quanto maior o percentual de caixa compondo o ativo circulante da empresa, menor o risco de liquidez. O caixa é a primeira fonte de recursos para os pagamentos de curto prazo nas MPEs.

Dívidas de curto prazo (DCP): as dívidas de curto prazo reduzem a capacidade de pagamento da empresa e o aumento do risco de liquidez.

Empréstimos: esta variável verifica se, na atualidade, a empresa possui empréstimos para pagamento das dívidas de curto prazo, atribuindo-se valor 0 para não-possui e 1 para possui. Esta variável mede a propensão do empresário a assumir riscos, bem como seu acesso a fontes externas de financiamento. Esperava-se que sua presença estivesse associada à maior risco de liquidez, em virtude, por exemplo, do custo relativamente alto deste recurso.

Falta de capital de giro: também se trata de variável qualitativa do tipo dummy, a qual se atribuiu 0 = não falta de capital de giro e 1 = falta capital de giro. Esta variável buscou questionar diretamente se o empresário tem problema de liquidez. 
Utilização de modelos de regressão logística para a previsão de risco de liquidez em micro e pequenas empresas

Lucas Maia dos Santos, Marco Aurélio Marques Ferreira, Evandro Rodrigues de Faria

Relatórios financeiros (RT): diz respeito à elaboração, pelas empresas ou contadores, e utilização de relatórios financeiros. Esperava-se que empresas habituadas ao uso destes relatórios fossem, em geral, mais prudentes, não incorrendo em problemas de capital de giro. Sabe-se que grande parte dos empresários possui dificuldades em relações a questões contábeis, todavia relatórios financeiros são de grande importância para prever necessidades de capital e monitorar a saúde financeira da empresa.

Controle de caixa: variável qualitativa do tipo dummy, a qual se atribuiu $0=$ não faz controle de caixa e 1 = faz controle de caixa. Associa-se maior risco de liquidez para aqueles que não procedem ao controle de caixa.

Pagamento adiantado: variável qualitativa do tipo dummy, a qual se atribuiu $0=$ não faz pagamento adiantado e 1 = faz pagamento adiantado. Associa-se maior risco de liquidez para aqueles que fazem qualquer pagamento adiantado, pois economizam com os descontos obtidos, bem como, demonstra a presença de recursos excedentes em caixa.

Conhecimento sobre administração financeira: escala de 1 a 5 , verificando o conhecimento do empresário de algumas terminologias financeiras. Supõe-se que o empresário com maior conhecimento está associado a um menor risco de liquidez.

Retiradas de caixa para gastos pessoais: variável qualitativa do tipo dummy, na qual se atribuiu 0 = não faz retiradas de caixa e 1 = faz retiradas de caixa. Associase maior risco de liquidez para aqueles que fazem retiradas de caixa para custear gastos pessoais, em decorrência da redução de recursos disponível para pagamento de despesas correntes.

Sexo do proprietário (SX): trata-se de variável qualitativa do tipo dummy, a qual se atribuiu $0=$ masculino e $1=$ feminino. Não se criaram expectativas em relação a esta variável, todavia optou-se por seu uso em razão do aumento da participação de mulheres nas atividades gerenciais.

Tempo de Experiência do proprietário (EX): refere-se ao total de anos dedicados pelo proprietário ao ramo em que atua. Era esperado desta variável grande relação com o problema em questão, levando-se em conta a contribuição do tempo 
Utilização de modelos de regressão logística para a previsão de risco de liquidez em micro e pequenas empresas

Lucas Maia dos Santos, Marco Aurélio Marques Ferreira, Evandro Rodrigues de Faria

para aquisição de conhecimentos acerca do mercado por parte do empresário. Segundo pesquisa SEBRAE (2007), conhecimento acerca do mercado é, para 55\% dos empresários, um dos fatores mais importantes para o sucesso de uma empresa.

Ciclo de caixa: refere-se ao tempo de imobilização do caixa. Assim, quanto maior o ciclo de caixa, maior é o risco de liquidez da empresa devido à imobilização financeira.

Por fim, cabe ressaltar que a inclusão de um modelo estatístico de probabilidade neste estudo visa identificar o impacto das variáveis associadas ao problema de capital de giro nas micro e pequenas empresas da cidade de Viçosa-MG.

\section{RESULTADOS}

A Tabela 1 informa como seriam classificados os indivíduos caso o modelo se deixasse guiar apenas pela situação em que se enquadra a maioria dos casos observados. Assim, agindo conforme explicado na metodologia para construção da variável dependente, a amostra foi dividida em dois grupos: 98 casos foram classificados no grupo sem risco de liquidez e 74 casos no grupo de risco de liquidez. De acordo com a Tabela 1, o modelo classificaria corretamente as empresas que não possuem risco de liquidez. Nesse caso, o percentual geral de acerto nas classificações seria de aproximadamente $57 \%$. Esse quadro de classificação anterior à análise atua, portanto, como uma referência para avaliar a eficácia do modelo quando ele passa a operar com as variáveis independentes para predizer a que grupo pertence certo indivíduo.

Tabela 1: Classificação inicial dos grupos antes da aplicação do modelo logit

\begin{tabular}{|c|c|c|c|c|}
\hline & & \multicolumn{2}{|c|}{ Risco de liquidez } & \multirow{2}{*}{$\begin{array}{c}\% \\
\text { Não }\end{array}$} \\
\hline & & Não & Sim & \\
\hline \multirow[t]{2}{*}{ Risco de liquidez } & Não & 98 & 0 & 100,0 \\
\hline & Sim & 74 & 0 & 0,0 \\
\hline \multicolumn{2}{|l|}{ Porcentagem total } & & & 57,0 \\
\hline
\end{tabular}

Fonte: Resultado da pesquisa 
Utilização de modelos de regressão logística para a previsão de risco de liquidez em micro e pequenas empresas

Lucas Maia dos Santos, Marco Aurélio Marques Ferreira, Evandro Rodrigues de Faria

A Tabela 2 exibe o quadro da estatística Wald, que nesse primeiro momento está avaliando a significância da constante incluída na função de regressão. Do exposto, conclui-se que seria conveniente formular predições em função desse critério de classificação, visto que o nível de significância foi de 6,8\%. O objetivo dessa estatística foi fornecer uma base de comparação que permita verificar se as variáveis independentes podem melhorar a qualidade das predições. É importante considerar que sem incluí-las no modelo já se alcança um nível de acerto aproximado de $57 \%$ na classificação dos casos. Acrescentando-as, espera-se um percentual mais elevado.

Tabela 2: Estatística de Wald para a constante da função de regressão

\begin{tabular}{|l|c|c|c|c|}
\hline & B & Erro-padrão. & Wald & Significância. \\
\hline Constante & $-0,281$ & 0,154 & 3,327 & 0,068 \\
\hline
\end{tabular}

Fonte: Resultado da pesquisa

Como evidencia a Tabela 3, uma das primeiras providências é testar a validade do modelo como um todo. Na regressão linear esse procedimento é realizado por meio da estatística $F$, cujo objetivo é testar a hipótese de que todos os coeficientes da equação são nulos. Como se sabe, a confirmação dessa hipótese sugere que o modelo não serve para a estimação de valores para a variável dependente em função de valores conhecidos das independentes, porque nesse caso nenhum dos coeficientes seria significativamente diferente de zero.

O modelo Chi-square testa a hipótese de que todos os coeficientes da equação logística são nulos. O valor do modelo, como mostrado na Tabela 3 foi de 133,47. O Likelihood (-2LL) e a diferença entre os valores iniciais e finais desse indicador expressam a capacidade preditiva do modelo. Esse número corresponde à diferença entre o valor de -2LL obtido quando se inclui apenas a constante no modelo e o -2LL calculado após a inclusão de todas as variáveis independentes. Com a inclusão das variáveis preditoras, espera-se que o Likelihood Value sofra uma redução estatisticamente significativa. De acordo com as significâncias das estatísticas demonstradas na Tabela 3, pode-se concluir que pelo menos um dos coeficientes da regressão logística é diferente de zero. Portanto, pode-se rejeitar a hipótese de que 

Utilização de modelos de regressão logística para a previsão de risco de liquidez em micro e
pequenas empresas

Lucas Maia dos Santos, Marco Aurélio Marques Ferreira, Evandro Rodrigues de Faria

todos os parâmetros estimados são nulos. Em outras palavras, pode-se afirmar que eles contribuem para melhorar a qualidade das predições.

Tabela 3: Testes de Omnibus

\begin{tabular}{|l|c|c|c|}
\hline & Chi-square & Grau de liberdade & Significância \\
\hline Step & 133,477 & 37 & 0,000 \\
\hline Block & 133,477 & 37 & 0,000 \\
\hline Model & 133,477 & 37 & 0,000 \\
\hline
\end{tabular}

Fonte: Resultado da pesquisa

Na Tabela 4, os testes Cox \& Snell e Nagelkerke são considerados pseudos- $R^{2}$. Eles procuram indicar a proporção das variações ocorridas no log da razão de chance que é explicada pelo conjunto das variáveis independentes. Assim, o Cox \& Snell está indicando que $54 \%$ das variações ocorridas no log da razão de chance são explicadas pelo conjunto das variáveis independentes. Com significado semelhante ao coeficiente de determinação, o Nagelkerke considera que o modelo é capaz de explicar $72,4 \%$ das variações registradas na variável dependente.

Tabela 4: Testes de Cox Snell e Nagelkerke

\begin{tabular}{|c|c|c|}
\hline $\mathbf{- 2}$ Log likelihood & Cox \& Snell R Square & Nagelkerke R Square \\
\hline 101,606 & 0,540 & 0,724 \\
\hline
\end{tabular}

Fonte: Resultado da pesquisa

$\mathrm{Na}$ Tabela 5, tem-se o indicador denominado teste Hosmer e Lemeshow. Tratase de um teste Qui-quadrado, cujo objetivo é testar a hipótese de que não há diferenças significativas entre os resultados preditos pelo modelo e os observados.

Seguindo uma distribuição Qui-quadrado, o cálculo leva a uma estatística de 8,227 e um nível de significância de $41,2 \%$. Isso indica que os valores preditos não são significativamente diferentes dos observados. Como o teste pretende aceitar a hipótese de que não existem diferenças entre valores preditos e observados, tem se mais um indício que o modelo pode ser utilizado para estimar a probabilidade de risco de liquidez em função das variáveis independentes. 
Utilização de modelos de regressão logística para a previsão de risco de liquidez em micro e pequenas empresas Lucas Maia dos Santos, Marco Aurélio Marques Ferreira, Evandro Rodrigues de Faria

Tabela 5: Teste de Hosmer and Lemeshow

\begin{tabular}{|c|c|c|}
\hline Chi-square & df & Sig. \\
\hline 8,227 & 8 & 0,412 \\
\hline
\end{tabular}

Fonte: Resultado da pesquisa

Sem incluir as variáveis independentes, o percentual de acertos era de $57 \%$. Como o quadro mostra, incluindo as variáveis independentes o modelo passa a ter um percentual de acerto de $87,2 \%$ com um nível de confiança de 10\%. Assim, estatisticamente, é viável incluir as variáveis no modelo.

Tabela 6: Classificação do modelo de regressão logística

\begin{tabular}{|c|c|c|c|c|}
\hline \multicolumn{2}{|l|}{} & \multicolumn{2}{c|}{ Risco de liquidez } & \% \\
\hline \multicolumn{2}{|l|}{} & Não & Sim & Não \\
\hline Risco de liquidez & Não & 89 & 9 & 90,8 \\
\hline \multicolumn{2}{|c|}{ Classificação total } & 13 & 61 & 82,4 \\
\hline \multicolumn{2}{|c|}{ Sim } & & & 87,2 \\
\hline
\end{tabular}

Fonte: Resultado da pesquisa

Como visto, todos os testes sugerem que, de forma geral, o modelo pode ser utilizado para estimar a probabilidade da empresa ter ou não risco de liquidez. Resta ainda verificar a significância de cada coeficiente em particular. Afinal, é necessário verificar se cada um deles realmente pode ser utilizado como estimador de probabilidades. Para tanto, recorre-se novamente à estatística Wald. Trata-se de um mecanismo equivalente ao teste $\mathrm{T}$, cujo objetivo é testar a hipótese nula de que um determinado coeficiente não é significativamente diferente de zero.

Como a variável independente tem apenas um grau de liberdade, para cada coeficiente procede-se ao seguinte cálculo:

Wald $=\left(\frac{b}{S \cdot E}\right)^{2}$

Equação 2

onde,

b simboliza o coeficiente de uma variável incluída no modelo

S.E, o erro padrão a ele associado. 
Utilização de modelos de regressão logística para a previsão de risco de liquidez em micro e pequenas empresas

Lucas Maia dos Santos, Marco Aurélio Marques Ferreira, Evandro Rodrigues de Faria

Como se observa na Tabela 7, nem todas as variáveis podem ser aproveitadas na composição do modelo, já que algumas possuem o coeficiente não diferente de zero, por causa de um nível de significância maior que 10\%. Dentre todas as variáveis utilizadas apenas as variáveis 'número de sócios', 'vendas a prazo' e 'empréstimos' mostraram coeficientes estatisticamente diferentes de zero para compor a função de probabilidade. Em outras palavras, pode-se afirmar que cada um deles exerce efeito sobre a probabilidade de uma empresa ter risco de liquidez pelo menos a um nível de significância de $10 \%$.

Observa-se na Tabela 7 que uma variação positiva para o número de sócios aumenta o log de probabilidade de risco de liquidez. Este resultado segue na contramão da explicação sobre esta variável, na metodologia deste estudo. Esperavase que esta variável tivesse um coeficiente negativo, ou seja, sua variação positiva reduziria o risco de liquidez.

Observa-se ainda na Tabela 7, por causa do coeficiente negativo, que uma variação positiva nas vendas à prazo aumente o log de probabilidade de risco de liquidez. Esperava-se o oposto, visto que o aumento das vendas a prazo aumenta o risco de inadimplência dos clientes que pode provocar falta de caixa.

Por fim, observa-se que a presença de empréstimos para pagamento de dívidas correntes aumenta a probabilidade de risco de liquidez, como esperado. Sabe-se que a necessidade de empréstimo para pagamento de dívidas de curto prazo, evidencia uma falta de caixa na empresa. 
Utilização de modelos de regressão logística para a previsão de risco de liquidez em micro e pequenas empresas Lucas Maia dos Santos, Marco Aurélio Marques Ferreira, Evandro Rodrigues de Faria

Tabela 7 - Variáveis na equação

\begin{tabular}{|c|c|c|c|c|}
\hline & B & S.E. & Wald & Significância. \\
\hline Tempo de existência & 0,020 & 0,032 & 0,397 & 0,529 \\
\hline Números de empregados & $-0,080$ & 0,104 & 0,592 & 0,442 \\
\hline Número de sócios & $\mathbf{0 , 8 7 9}$ & $\mathbf{0 , 4 9 6}$ & $\mathbf{3 , 1 4 5}$ & $\mathbf{0 , 0 7 6}$ \\
\hline Setor de atividade & $-0,279$ & 0,724 & 0,149 & 0,700 \\
\hline Percentual de capital próprio & 0,070 & 0,095 & 0,546 & 0,460 \\
\hline Presença de investimentos & 0,720 & 0,753 & 0,915 & 0,339 \\
\hline Faturamento mensal & 0,000 & 0,000 & 0,894 & 0,344 \\
\hline Vendas á prazo & $-\mathbf{0 , 0 9 0}$ & $\mathbf{0 , 0 5 3}$ & $\mathbf{2 , 8 1 8}$ & $\mathbf{0 , 0 9 3}$ \\
\hline Prazo médio de recebimento & 0,022 &, 020 & 1,329 & 0,249 \\
\hline Prazo médio pagamento & $-0,013$ & 0,031 & 0,176 & 0,675 \\
\hline Percentual de caixa & 16,657 & 23,739 & 0,492 & 0,483 \\
\hline Dividas de curto prazo & $-0,294$ & 0,275 & 1,142 & 0,285 \\
\hline Empréstimos & $\mathbf{3 , 5 8 9}$ & $\mathbf{1 , 4 5 7}$ & $\mathbf{6 , 0 7 2}$ & $\mathbf{0 , 0 1 4}$ \\
\hline Falta de capital de giro & 143,965 & 40193,355 & 0,000 & 0,997 \\
\hline Relatório financeiro & 0,186 & 0,697 & 0,071 & 0,790 \\
\hline Controle de caixa & 0,272 & 0,635 & 0,184 & 0,668 \\
\hline Pagamentos adiantados & 0,515 & 0,623 & 0,683 & 0,409 \\
\hline Conhecimento em administração financeira & $-0,485$ & 0,401 & 1,464 & 0,226 \\
\hline Retiradas de caixa para gastos pessoais & $-0,035$ & 0,703 & 0,002 & 0,961 \\
\hline Sexo & $-0,284$ & 0,672 & 0,179 & 0,672 \\
\hline Tempo de experiência & 0,026 & 0,052 & 0,251 & 0,616 \\
\hline Ciclo de caixa & $-0,019$ & 0,027 & 0,459 & 0,498 \\
\hline Constante & $-1677,026$ & 13113,930 & 0,016 & 0,898 \\
\hline
\end{tabular}

Fonte: Resultado da pesquisa

O Gráfico 1 mostra como foram classificadas cada empresa da amostra na previsão. Ao observar os pontos no gráfico, percebem-se quais indivíduos foram classificados corretamente ou não, dentro da previsão. 
Utilização de modelos de regressão logística para a previsão de risco de liquidez em micro e pequenas empresas

Lucas Maia dos Santos, Marco Aurélio Marques Ferreira, Evandro Rodrigues de Faria

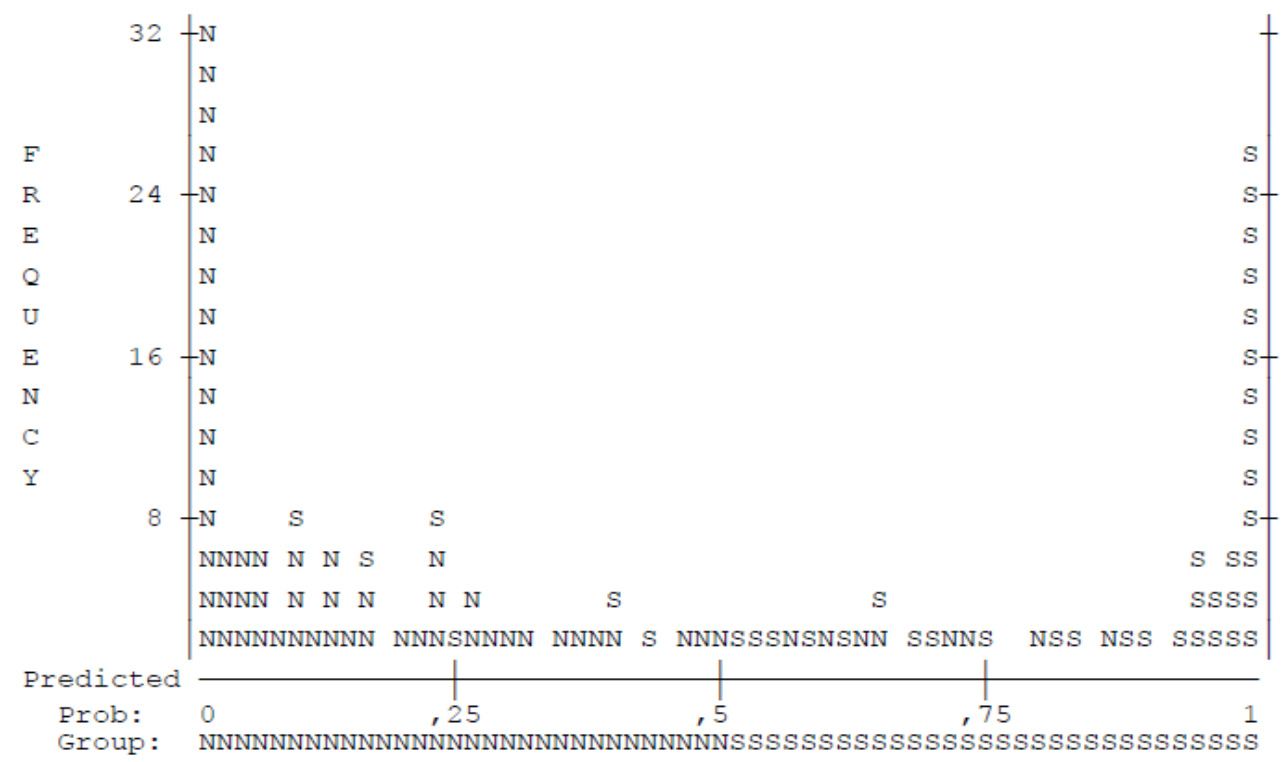

Símbolos: $\mathrm{N}$ - Sem risco de liquidez $\quad \mathrm{S}$ - Risco de liquidez

Cada símbolo representa dois casos

Gráfico 1 - Probabilidade dos casos

Submetido a todos os testes, verifica-se que o modelo está estatisticamente apto a ser utilizado na solução do problema de investigar a probabilidade de previsão de risco de liquidez. Desta forma, pode-se esboçar a equação da regressão logística com boa margem de segurança.

Fica a seguinte função de regressão:

$P(y)=\frac{1}{1+e^{-(0,879 N S-0,090 V P+3,589 E P)}}$

Equação 3

Como se verifica na regressão linear, cada coeficiente deve ser interpretado como estimativa do efeito que uma variável independente produz sobre a dependente quando as demais se mantêm inalteradas. Entretanto, não se pode esquecer que o modelo logístico é expresso em termos de logaritmos da razão de chance ou logit. Assim, cada coeficiente deve ser interpretado como o efeito que uma variação unitária sofrida pela variável independente tende a produzir sobre o logaritmo da razão de chance. O sinal do coeficiente é que vai determinar a direção da mudança, que pode 
Utilização de modelos de regressão logística para a previsão de risco de liquidez em micro e pequenas empresas

Lucas Maia dos Santos, Marco Aurélio Marques Ferreira, Evandro Rodrigues de Faria

ser aumentativa ou diminutiva. Já o efeito de cada coeficiente sobre a probabilidade é de natureza multiplicativa e vai depender do nível em que ela se encontrar.

\section{CONSIDERAÇÕES FINAIS}

Este estudo evidenciou a importância da gestão financeira de curto prazo nas MPEs da região em estudo, tendo em vista que problemas de liquidez são uma das principais causas de seu fracasso. É sabido que a saúde financeira da organização é determinada por inúmeras variáveis, especialmente em micro e pequenas empresas, em que as ferramentas de gestão se misturam à personalidade do proprietário, tornando ainda mais complexo qualquer tipo de estudo.

A classificação das empresas analisadas em dois grupos mostrou-se eficiente para demonstrar a existência de variáveis condicionantes do risco de liquidez nas MPEs. Número de sócios, vendas a prazo e empréstimos são alguns dos fatores que mais podem determinar o risco de liquidez nas MPEs na área em estudo, de acordo com a regressão logística.

Apesar de todos os testes mostrarem-se significantes para introdução das diversas variáveis independentes na previsão de risco de liquidez, no final, apenas três resultaram em coeficientes estatisticamente diferentes de zero.

Desta forma, problemas de liquidez podem ser evitados por diversas práticas de gerenciamento e sua previsão é de extrema importância, levando em consideração um conjunto de empresas semelhantes. Sugere-se a aplicação deste modelo em outras amostras e em maior abrangência regional, dada a importância que a previsão deste risco tem para a sobrevivência destas empresas.

\section{REFERÊNCIAS}

BILÉSSIMO, L. D. (2002). Instrumento para diagnóstico da expectativa de sucesso da micro e pequena empresa brasileira. 160 f. (Dissertação de Mestrado). Universidade Federal de Santa Catarina, Florianópolis. Disponível em: <http://teses.eps.ufsc.br/defesa/pdf/5440.pdf>. Acesso em: 25/jun/2006. 

Utilização de modelos de regressão logística para a previsão de risco de liquidez em micro e
pequenas empresas

Lucas Maia dos Santos, Marco Aurélio Marques Ferreira, Evandro Rodrigues de Faria

BOSMA, N.; JONES, K.; AUTIO, E.; LEVIE, J. (2008). Global Entrepreneurship Monitor. Babson College London Business School. Babson Park, MA, US London, UK, p.66.

BRAGA, R.; NOSSA, V.; MARQUES, J.A.V.C. (2004). Uma proposta para a análise integrada da liquidez e rentabilidade das empresas. Revista Contabilidade \& Finanças, edição especial, p. 51-64.

CHÉR, R. (1990). A gerência das pequenas e médias empresas: o que saber para administrá-las. São Paulo: Maltese.

GAZZONI, E. I. (2003). Fluxo de caixa- ferramenta de controle financeiro para pequena empresa. 96 f. (Dissertação de Mestrado). Universidade Federal de Santa Catarina, Florianópolis. Disponível em: <http://teses.eps.ufsc.br/defesa/pdf/9318.pdf>. Acesso em: 26/jun/2006.

HOPP, J.C.; LEITE. H.P. (1989). O mito da liquidez. Revista de Administração de Empresas, São Paulo, v. 29, n.4, p. 63-69, out / dez.

KASSAI, J.R.; KASSAI, S. (2001). Balanço perguntado: solução para as pequenas empresas. In: Congresso Brasileiro de Custos, 8, Rio Grande do Sul. Anais...São Leopoldo.

LEMES JUNIOR, A. C. B., RIGO, C. M., et al. (2005). Administração financeira: princípios, fundamentos e práticas trabalhistas. Rio de Janeiro: Elsevier. 547 p.

MATIAS, G. A.; LOPES JR., F. (2002). Administração financeira nas empresas de pequeno porte. São Paulo: Manole.

MONTEIRO, A. A. S. M. (2003). Fluxos de caixa e capital de giro: uma adaptação do modelo de Fleuriet. In: Seminário de Contabilidade e Controladoria, 2, São Paulo. Anais... São Paulo: FEA-USP. Disponível em: <http://www.eac.fea.usp.br> Acesso em: 4/Maio/2003.

MORAES, E. G.; RHODEN, M. I. S. (2005). Determinantes da estrutura de capital das empresas listadas na Bovespa. In: ENCONTRO NACIONAL DOS PROGRAMAS DE PÓS-GRADUAÇÃO EM ADMINISTRAÇÃO, 29, Brasília, Anais...Brasília: EnANPAD.

MYERS, S.C. (1984). The capital structure puzzle. Journal of Finance, Chicago: American Finance Association, v.39, n.3.

MYERS, S.C.; MAJLUF, N. (1984). Corporate financing and investment decisions when firms have informations that investors do not have. Journal of Financial Economics, vol. 13 , p. $187-221$. 

Utilização de modelos de regressão logística para a previsão de risco de liquidez em micro e
pequenas empresas

Lucas Maia dos Santos, Marco Aurélio Marques Ferreira, Evandro Rodrigues de Faria

NEVES, J.A.D.; PESSOA, R.W.A.P. (2006). Causas da mortalidade de micro e pequenas empresas: o caso das lojas de um Shopping Center. Revista Organizações em contexto, n.4, p. 165-195.

PASSOS, C. A. K.; FELIX, J.C; GRECO, S. M. S.S. (2008). Empreendedorismo no Brasil: 2007. Instituto Brasileiro de Qualidade e Produtividade, p.171.

PEREIRA FILHO, A. D. (1998). O modelo dinâmico de gestão financeira de empresa: procedimentos de operacionalização. Revista Contabilidade Vista \& Revista, Belo Horizonte, v. 9, n.4, p.12-22.

RESNIK, P. (1990). A bíblia da pequena empresa: como iniciar com segurança sua pequena empresa e ser muito bem sucedido. São Paulo: McGraw-Hill.

SERVIÇO BRASILEIRO DE APOIO ÀS MICRO E PEQUENAS EMPRESAS -SEBRAE. (2005). Boletim estatístico de micro e pequenas empresas. Brasília. Disponível em: <http://201.2.114.147/bds/BDS.nsf/03DE0485DB219CDE0325701B004CBD01/\$File/NT 000A8E66.pdf>. Acesso em: 20/fev/2006.

$\begin{array}{lll} & & \\ \text { e pequenas } & \text { (2007). Fatores condicionantes e taxa de mortalidade das micro } \\ \text { empresas. VOX POPULI. Disponível em: }\end{array}$ <http://www.biblioteca.sebrae.com.br/bds/BDS.nsf/8F5BDE79736CB99483257447006C BAD3/\$File/NT00037936.pdf>. Acesso em 18/jan/2008.

Silva, J. P. (2006). Análise financeira das empresas. São Paulo: Atlas.

SOUZA, A. F. (1997). Estratégia, crescimento e a administração do capital de giro. Caderno de Pesquisa em Administração. Programa de Pós-Graduação, FEA, USP, v.2, p.12.

SOUZA, R.M. (2007). Avaliação de custo, volume e lucro em micro e pequenas empresas comerciais: um estudo de caso. 107 f. (Dissertação de Mestrado). Universidade Federal de Itajubá, Itajubá.

Data de Submissão: 22/07/2009

Data de Aceite: 15/10/2009 